\title{
Epidemic Model Analysis of Covid-19
}

\author{
Dayat Hidayat ${ }^{*}$, Edwin Setiawan Nugraha ${ }^{2}$ \\ ${ }^{1}$ Mathematics Department, Universitas Negeri Surabaya, Surabaya, Indonesia \\ ${ }^{2}$ Faculty of Business, Study Program of Actuarial Science, President University, Cikarang, Bekasi, Indonesia
}

\begin{abstract}
Covid-19 is a very extraordinary case not only in one country but all countries in the world. The number of deaths caused by Covid-19 is very large and the rate of spread of this disease is very high and fast. In this paper, we perform an analysis of a covid-19 epidemic model. This model is a development of the SEIR model in general which is equipped with a Quarantine (Q), Fatality (F) compartment, and there is a separation between detected and undetected infected people (I). Our analysis shows that there are two equilibria, namely, disease free equilibrium and endemic equilibrium. by using, Lyapunov function, we demonstrated that disease free is globally asymptotically stable if $R 0<1$, and disease-free becomes unstable if $R 0>1$. This result reveal that the intervention of infection rate and quarantine process are important to control and achieve global stability of disease-free equilibrium
\end{abstract}

Keywords: Covid-19, Epidemic Model, SEIR

\section{Introduction}

At present, all countries in the world focus on pandemic diseases, namely the Corona virus disease 2019 or COVID-19 caused by severe acute respiratory syndrome corona virus 2 (SARS-Cov-2). This virus was first identified in late December 2019 in Wuhan, China. Since early 2020, COVID-19 has begun to spread to various parts of the world and caused millions of people to become infected. Till September 09, 2021 there were $223,472,097$ cases and 4,611,333 deaths worldwide. Indonesia was recorded 4,147,365 cases with 137,782 deaths [1]. According to WHO [2], people can be suffered covid-19 by people who have the virus. The disease spread through small droplets from nose and mouth when a person coughs, sneezes, or speaks. People can be infected the disease when they breath in these droplets from infected person or indirectly while people touch some objects or surfaces that droplet landed.

The rapid spread of the virus has led countries to think about effective ways to reduce mortality. Because no cure method has yet been found for this virus, planning is needed to control the spread of the disease. Therefore, estimating the total confirmed cases and predicting the possibility of new cases that emerge becomes very important in making strategic policies in the health sector. One tool that can be used in predicting the number of cases that will appear based on data or parameters known so as to be able to make predictions

\footnotetext{
* Corresponding author : dayathidayat@unesa.ac.id
}

of ways to deal with outbreaks is by mathematical modeling.

There are many mathematical models that discuss the spread of covid-19. Some of them are modeling using statistical approaches. For instance, Ceylan [3] investigated epidemiologcal trend of covid-19 by using time series models with Auto Regressive Integrated Moving Average (ARIMA) to find the trends of the outbreak in Italy, Spain, and France. In line with [3], Chintalapudi et al. [4] applied ARIMA models in purpose for forcasting of infected people and highlighted the importance of country lockdown and self-isolation. In addition, other researcher even analyzed stochastic models with Monte-Carlo simulations using MATLAB for estimating the impact of social distancing and lockdown in India [5]. In the other hand, some of researchers also make the development of mathematical models using differential equations, namely the general SEIR epidemic model [6]. Moreover, Mandal et al. [7] investigated the dynamical behavior of development of SEIR models where homestead isolation, quarantine, and optimal control are considered. In addition, He et al. [8] also developed SEIR models with taking into account of some general control strategies, such as hospital, quarantine, and external input to show the evolution of the epidemic in Hubai Province. Several studies of development SEIR models also considering detected and undetected asymptomatic cases $[9,10]$. For instance, Aldila et al [10] Formulated a modified SEIR models considering asymptomatic individuals to investigate the effect of 
rapid testing and social distancing in controlling the spread of covid-19 in Jakarta, Indonesia.

In this study, we developed the SEIR model by involving the home-stead isolation, detected and undetected asymptomatic cases, and fatality cases. Basic reproductive ratio is obtained and stability analysis of the equilibria is shown. Moreover, we do an in-depth mathematical analysis of the global stability of its equilibrium point through Lyapunov function.

\section{Model Formulation}

Here, we contruct the epidemic model of covid-19 which is divided into seven compartments, i.e., susceptible (S), exposed (E), detectable Infected $\left(I_{1}\right)$, undetectable Infected $\left(I_{2}\right)$, Quarantine $(\mathrm{Q})$, Recovered (R), Fatality (F)

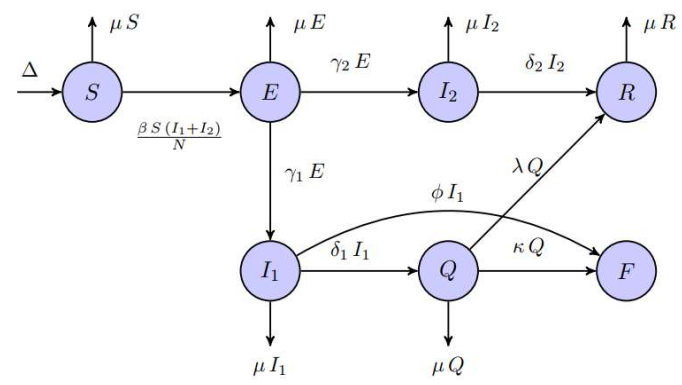

Fig. 1. Covid 19 Transmission Diagram

According to the description, we can express the model in the following differential equation system

$$
\begin{aligned}
\dot{S} & =\Lambda-\frac{\beta S}{N}\left(I_{1}+I_{2}\right)-\mu S \\
\dot{E} & =\frac{\beta S}{N}\left(I_{1}+I_{2}\right)-\left(\gamma_{1}+\gamma_{2}+\mu\right) E \\
\dot{I} & =\gamma_{1} E-\left(\delta_{1}+\mu\right) I_{1}-\phi I_{1} \\
\dot{I}_{2} & =\gamma_{2} E-\left(\delta_{2}+\mu\right) I_{2} \\
\dot{Q} & =\delta_{1} I_{1}-\lambda Q-\kappa Q-\mu Q \\
\dot{F} & =\kappa Q+\phi I_{1} \\
\dot{R} & =\lambda Q+\delta_{2} I_{2}-\mu R
\end{aligned}
$$

Table 1. List of parameters of the model

\begin{tabular}{|l|l|}
\hline $\begin{array}{l}\text { Paramete } \\
\text { rs }\end{array}$ & Description \\
\hline$\beta$ & The infection rates \\
$\omega$ & The latency coefficient \\
$\gamma$ & The removal rate \\
$m$ & Disinfection and ventilation efficiency \\
$\delta$ & The vaccination coefficient \\
$\psi$ & The isolation rate \\
$\Delta$ & The recruitment rates \\
$\mu$ & The natural death rate \\
\hline
\end{tabular}

\section{Basic Reproduction Number}

Disease Free Equilibrium

$$
\begin{array}{r}
D F E=(E=0 ; F=0 ; Q=0 ; R=0 ; S \\
\left.=\Delta / \mu ; I_{1}=0 ; I_{2}=0\right)
\end{array}
$$

Matriks F:

$$
F=\left[\begin{array}{ccc}
0 & \frac{\beta \Delta}{\mu N} & \frac{\beta \Delta}{\mu N} \\
\gamma_{1} & 0 & 0 \\
\gamma_{2} & 0 & 0
\end{array}\right]
$$

Matrix V:

$$
V=\left[\begin{array}{ccc}
-\gamma_{1}-\gamma_{2}-\mu & 0 & 0 \\
0 & -\mu-\delta_{1} & 0 \\
0 & 0 & -\mu-\delta_{2}
\end{array}\right]
$$

Matrix NGM:

$$
N G M=\left[\begin{array}{ccc}
0 & \frac{\beta \Delta}{\mu N\left(\mu+\delta_{1}\right)} & \frac{\beta \Delta}{\mu N\left(\mu+\delta_{2}\right)} \\
\frac{\gamma_{1}}{\gamma_{1}+\gamma_{2}+\mu} & 0 & 0 \\
\frac{\gamma_{2}}{\gamma_{1}+\gamma_{2}+\mu} & 0 & 0
\end{array}\right]
$$

Basic reproduction number

$$
\bar{R}_{0}=\sqrt{\frac{\Delta \beta\left(\gamma_{1} \mu+\gamma_{2} \mu+\gamma_{2} \delta_{1}+\delta_{2} \gamma_{1}\right)}{\left(\gamma_{1}+\gamma_{2}+\mu\right) \mu N\left(\mu+\delta_{1}\right)\left(\mu+\delta_{2}\right)}}
$$

Equivalent threshold parameters:

$$
R_{0}=\bar{R}_{0}^{2}
$$

\section{Disease Free Equilibrium and Local Stability}

Evaluation of Jacobian Matrix at $E_{0}$

$J(D F E)=\left[\begin{array}{ccccccc}-\mu & 0 & -\frac{\Delta \beta}{N \mu} & -\frac{\Delta \beta}{N \mu} & 0 & 0 & 0 \\ 0 & -\gamma_{1}-\gamma_{2}-\mu & \frac{\Delta \beta}{N \mu} & \frac{\Delta \beta}{N \mu} & 0 & 0 & 0 \\ 0 & \gamma_{1} & -\mu-\delta_{1} & 0 & 0 & 0 & 0 \\ 0 & \gamma_{2} & 0 & -\mu-\delta_{2} & 0 & 0 & 0 \\ 0 & 0 & \delta_{1} & 0 & -\kappa-\lambda-\mu & 0 & 0 \\ 0 & 0 & 0 & \delta_{2} & \lambda & -\mu & 0 \\ 0 & 0 & 0 & 0 & \kappa & 0 & -\mu\end{array}\right]$

with characteristic polynomials:

$p(\lambda)=(\lambda+\mu)^{3}(2 \lambda+\mu+\kappa)\left(a_{3} \lambda^{3}+a_{2} \lambda^{2}+a_{1} \lambda+a_{0}\right)$

were,

$$
a_{3}=1
$$$$
a_{2}=\left(3 \mu+\delta_{1}+\delta_{2}+\gamma_{1}+\gamma_{2}\right)
$$

$a_{1}=-\Delta \beta\left(\gamma_{1}+\gamma_{2}\right) /(\mu N)+\left(\left(2 \mu+\delta_{1}+\delta_{2}\right)\left(\gamma_{1}+\gamma_{2}\right)+3 \mu^{2}+2 \mu \delta_{1}+2 \mu \delta_{2}+\delta_{1} \delta_{2}\right)$ $a_{0}=\left(\gamma_{1}+\gamma_{2}+\mu\right)\left(\mu+\delta_{1}\right)\left(\mu+\delta_{2}\right)\left(1-R_{0}\right)$

Since the values of all parameter are positive, then $a_{3}$ and $a_{2}$ are positive. If $R_{0}<1$, this $a_{0}>0$.

Next, we need to show that al is positive. Consider

$R_{0}<1 \Longleftrightarrow \frac{\Delta \beta\left(\gamma_{1} \mu+\gamma_{2} \mu+\delta_{1} \gamma_{2}+\delta_{2} \gamma_{1}\right)}{\mu N\left(\gamma_{1}+\gamma_{2}+\mu\right)\left(\mu+\delta_{1}\right)\left(\mu+\delta_{2}\right)}<1$

And

$a_{1}>0 \Longleftrightarrow \frac{\Delta \beta\left(\gamma_{1}+\gamma_{2}\right)}{\mu N\left(\left(2 \mu+\delta_{1}+\delta_{2}\right)\left(\gamma_{1}+\gamma_{2}\right)+3 \mu^{2}+2 \mu \delta_{1}+2 \mu \delta_{2}+\delta_{1} \delta_{2}\right)}<1$.

Now, let

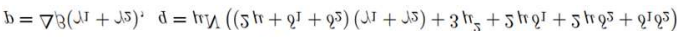

$r=\Delta \beta\left(\gamma_{1} \mu+\gamma_{2} \mu+\gamma_{2} \delta_{1}+\delta_{2} \gamma_{1}\right)$

$s=\mu N\left(\gamma_{1}+\gamma_{2}+\mu\right)\left(\mu+\delta_{1}\right)\left(\mu+\delta_{2}\right)$

where $r / s=R_{0}$, so that $p / q<R_{0}=r / s$.

$p s-q r=\Delta \beta\left(\gamma_{1} \mid+\gamma_{2}\right)\left(\mu N\left(\gamma_{1}+\gamma_{2}+\mu\right)\left(\mu+\delta_{1}\right)\left(\mu+\delta_{2}\right)\right)$

$-\Delta \beta\left(\gamma_{1} \mu+\gamma_{2} \mu+\gamma_{2} \delta_{1}+\delta_{2} \gamma_{1}\right)\left(\gamma_{1}+\gamma_{2}+\mu\right) \mu N\left(\mu+\delta_{1}\right)\left(\mu+\delta_{2}\right)$ $=-\mu N \beta \Delta\left(\left(\mu+\delta_{2}\right)^{2} \gamma_{1}^{2}+\left(\mu+\delta_{1}\right)^{2} \gamma_{2}^{2}+\left(\mu+\delta_{2}\right)^{2}\left(2 \mu+\delta_{1}\right) \gamma_{1}+\left(\mu+\delta_{1}\right)^{2}\left(2 \mu+\delta_{2}\right) \gamma_{2}\right.$ $\left.+\left(\left(\mu+\delta_{1}\right)^{2}+\left(\mu+\delta_{2}\right)^{2}\right) \gamma_{1} \gamma_{2}\right)$ 
The fact $R_{0}<1$ and $p q-r s<0$ this implies $a_{1}>0$.

$a_{2} a_{1}-a_{0}=\left(3 \mu+\delta_{1}+\delta_{2}+\gamma_{1}+\gamma_{2}\right)$

$\left(-\Delta \beta\left(\gamma_{1}+\gamma_{2}\right) /(\mu N)+\left(\left(2 \mu+\delta_{1}+\delta_{2}\right)\left(\gamma_{1}+\gamma_{2}\right)+3 \mu^{2}+2 \mu \delta_{1}+2 \mu \delta_{2}+\delta_{1} \delta_{2}\right)\right)$ $-\left(\gamma_{1}+\gamma_{2}+\mu\right)\left(\mu+\delta_{1}\right)\left(\mu+\delta_{2}\right)\left(1-R_{0}\right) \quad(8)$

$$
\begin{aligned}
& =-\frac{\Delta \beta\left(2 \gamma_{1} \mu+2 \gamma_{2} \mu+\delta_{1} \gamma_{1}+\delta_{2} \gamma_{2}+\gamma_{1}{ }^{2}+2 \gamma_{1} \gamma_{2}+\gamma_{2}{ }^{2}\right)}{\mu N} \\
& +\left(2 \mu+\delta_{2}+\gamma_{1}+\gamma_{2}\right)\left(2 \mu+\delta_{1}+\gamma_{1}+\gamma_{2}\right)\left(2 \mu+\delta_{1}+\delta_{2}\right)
\end{aligned}
$$

For case,

$$
R_{0}<1 \quad \Longleftrightarrow \quad \Delta \beta<\frac{\mu N\left(\mu+\gamma_{1}+\gamma_{2}\right)\left(\mu+\delta_{1}+\delta_{2}\right)}{\gamma_{1}\left(\mu+\delta_{2}\right)+\gamma_{2}\left(\mu+\delta_{1}\right)}
$$

If we substitute,

$$
\Delta \beta=\frac{\mu N\left(\mu+\gamma_{1}+\gamma_{2}\right)\left(\mu+\delta_{1}+\delta_{2}\right)}{\gamma_{1}\left(\mu+\delta_{2}\right)+\gamma_{2}\left(\mu+\delta_{1}\right)}
$$

to the equation [8] It follows,

$$
\begin{gathered}
a_{2} a_{1}-a_{0}>-\left(\gamma_{1}\left(2 \mu+\delta_{2}\right)+\gamma_{2}\left(2 \mu+\delta_{1}\right)+\left(\gamma_{1}+\gamma_{2}\right)^{2}\right) \frac{\left(\mu+\gamma_{1}+\gamma_{2}\right)\left(\mu+\delta_{1}+\delta_{2}\right)}{\gamma_{1}\left(\mu+\delta_{2}\right)+\gamma_{2}\left(\mu+\delta_{1}\right)} \\
+\left(2 \mu+\delta_{2}+\gamma_{1}+\gamma_{2}\right)\left(2 \mu+\delta_{1}+\gamma_{1}+\gamma_{2}\right)\left(2 \mu+\delta_{1}+\delta_{2}\right) \\
=K\left(\left(\mu+\delta_{2}\right)^{2} \gamma_{1}{ }^{2}+\gamma_{2}\left(\mu+\delta_{1}\right)^{2}\left(\gamma_{2}+\left(2 \mu+\delta_{2}\right)\right)\right. \\
\left.\gamma_{2} \gamma_{1}\left(\mu+\delta_{1}\right)^{2}+\gamma_{2} \gamma_{1} \delta_{1}^{2}+\gamma_{1}\left(\mu+\delta_{2}\right)^{2}\left(\gamma_{2}+\left(2 \mu+\delta_{1}\right)\right)\right)
\end{gathered}
$$

Where

$$
K=\left(\frac{3 \mu+\delta_{1}+\delta_{2}+\gamma_{1}+\gamma_{2}}{\gamma_{1} \mu+\gamma_{2} \mu+\delta_{1} \gamma_{2}+\delta_{2} \gamma_{1}}\right)
$$

Based on Routh Hurwitz Criteria, we conclude that all eigen values for factor cubic polynomial are negative. This complete the proof.

\section{Endemic And Local Stability}

Endemic point is $E N D=\left\{S^{*}, E^{*}, I_{1}^{*}, I_{2}^{*}, Q^{*}, R^{*}, F^{*}\right\}$ were

$$
\begin{gathered}
S^{*}=\frac{\delta N\left(\mu+\delta_{1}\right)\left(\mu+\delta_{2}\right)}{N \mu\left(\mu+\delta_{1}\right)\left(\mu+\delta_{2}\right)+\beta E^{*}\left(\gamma_{1}\left(\mu+\delta_{2}\right)+\gamma_{2}\left(\mu+\delta_{1}\right)\right)}, \\
I_{1}^{*}=\frac{\gamma_{1} E^{*}}{\mu+\delta_{1}}, I_{2}^{*}=\frac{\gamma_{2} E^{*}}{\mu+\delta_{2}}, Q^{*}=\frac{\gamma_{1} \delta_{1} E^{*}}{\left(\mu+\delta_{1}\right)(\kappa+\lambda+\mu)}, \\
R^{*}=\frac{E^{*}}{\mu}\left(\frac{\lambda \gamma_{1} \delta_{1}}{\left(\mu+\delta_{1}\right)(\kappa+\lambda+\mu)}+\frac{\gamma_{2} \delta_{2}}{\mu+\delta_{2}}\right), F^{*}=\frac{\kappa \gamma_{1} \delta_{1} E^{*}}{\mu\left(\mu+\delta_{1}\right)(\kappa+\lambda+\mu)} \\
E^{*}=\frac{N \mu\left(\mu+\delta_{1}\right)\left(\mu+\delta_{2}\right)\left(R_{0}-1\right)}{\beta\left(\gamma_{1} \mu+\gamma_{2} \mu+\delta_{1} \gamma_{2}+\delta_{2} \gamma_{1}\right)}
\end{gathered}
$$

Evaluate Jacobi Matrix on END:

$$
E N D=\left[\begin{array}{ccccccc}
-\frac{\beta\left(I_{1}^{+}+I_{2}^{*}\right)}{N}-\mu & 0 & -\frac{S^{*} \beta}{N} & -\frac{S^{*} \beta}{N} & 0 & 0 & 0 \\
\frac{\beta\left(I_{i}^{*}+I_{2}^{*}\right)}{N} & -\gamma_{1}-\gamma_{2}-\mu & \frac{S^{*} \beta}{N} & \frac{S^{*} \beta}{N} & 0 & 0 & 0 \\
0 & \gamma_{1} & -\mu-\delta_{1} & 0 & 0 & 0 & 0 \\
0 & \gamma_{2} & 0 & -\mu-\delta_{2} & 0 & 0 & 0 \\
0 & 0 & \delta_{1} & 0 & -\kappa-\lambda-\mu & 0 & 0 \\
0 & 0 & 0 & \delta_{2} & \lambda & -\mu & 0 \\
0 & 0 & 0 & 0 & \kappa & 0 & -\mu
\end{array}\right]
$$

with characteristic polynomials:

$p(\lambda) \mid=(\lambda+\mu)^{2}(2 \lambda+\mu+\kappa)\left(b_{4} \lambda^{4}+b_{3} \lambda^{3}+b_{2} \lambda^{2}+b_{1} \lambda+b_{0}\right)$

With $b_{4}=$

$b_{3}=\left(\gamma_{1}+\gamma_{2}+\delta_{1}+\delta_{2}+4 \mu\right)+\beta / N\left(I_{1}^{*}+I_{2}^{*}\right)$

$b_{2}=\left(2 \mu+\delta_{1}+\delta_{2}\right)\left(\gamma_{1}+\gamma_{2}+3 \mu\right)+\mu\left(\gamma_{1}+\gamma_{2}\right)+\delta_{1} \delta_{2}$

$\beta / N\left(3 \mu+\delta_{1}+\delta_{2}+\gamma_{1}+\gamma_{2}\right)\left(I_{1}^{*}+I_{2}^{*}\right)-S^{*} \beta / N\left(\gamma_{1}+\gamma_{2}\right)$

$b_{1}=\left(3 \mu^{2}+2 \mu \delta_{1}+2 \mu \delta_{2}+\delta_{1} \delta_{2}\right)\left(\gamma_{1}+\gamma_{2}\right)+\mu^{2}\left(4 \mu+3 \delta_{1}+3 \delta_{2}\right)+2 \mu \delta_{1} \delta_{2}$

$\beta / N\left(\left(2 \mu+\delta_{1}+\delta_{2}\right)\left(\gamma_{1}+\gamma_{2}\right)+\mu\left(3 \mu+2 \delta_{1}+2 \delta_{2}\right)+\delta_{1} \delta_{2}\right)\left(I_{1}^{*}+I_{2}^{*}\right)-S^{*} \beta / N\left(\gamma_{1}\left(2 \mu+\delta_{2}\right)+\gamma_{2}\left(2 \mu+\delta_{1}\right)\right)$

$b_{0}=\mu\left(\mu+\delta_{2}\right)\left(\mu+\delta_{1}\right)\left(\gamma_{1}+\gamma_{2}+\mu\right)+\beta / N\left(\gamma_{1}+\gamma_{2}+\mu\right)\left(\mu+\delta_{2}\right)\left(\mu+\delta_{1}\right)\left(I_{1}^{*}+I_{2}^{*}\right)$

$-\mu S^{*} \beta / N\left(\gamma_{1} \mu+\gamma_{2} \mu+\delta_{1} \gamma_{2}+\delta_{2} \gamma_{1}\right)$

Clearly that $b_{4}$, and $b_{3}$ are positive values. Since there are some positive and negative terms, then the sign of $b_{2}, b_{1}$, and $b_{0}$ cannot be able to determined directly. After substitute of $S^{*}$ to above equation, we have

$$
b_{2}=\beta / N\left(3 \mu+\delta_{1}+\delta_{2}+\gamma_{1}+\gamma_{2}\right)\left(I_{1}^{*}+I_{2}^{*}\right)
$$

$\frac{\left(\mu+\delta_{2}\right)\left(2 \mu+\delta_{2}\right) \gamma_{1}{ }^{2}+\left(\mu+\delta_{1}\right)\left(2 \mu+\delta_{1}\right) \gamma_{2}{ }^{2}+\left(4 \mu^{2}+3 \mu \delta_{1}+3 \mu \delta_{2}+\delta_{1}{ }^{2}+\delta_{2}{ }^{2}\right) \gamma_{2} \gamma_{1}}{\gamma_{1} \mu+\gamma_{2} \mu+\delta_{1} \gamma_{2}+\delta_{2} \gamma_{1}}$ $+\frac{\left(\mu+\delta_{2}\right)\left(5 \mu^{2}+2 \mu \delta_{1}+3 \mu \delta_{2}+\delta_{1} \delta_{2}\right) \gamma_{1}+\left(\mu+\delta_{1}\right)\left(5 \mu^{2}+3 \mu \delta_{1}+2 \mu \delta_{2}+\delta_{1} \delta_{2}\right) \gamma_{2}}{\gamma_{1} \mu+\gamma_{2} \mu+\delta_{1} \gamma_{2}+\delta_{2} \gamma_{1}}$

$b_{1}=\beta / N\left(\left(2 \mu+\delta_{1}+\delta_{2}\right)\left(\gamma_{1}+\gamma_{2}\right)+\mu\left(3 \mu+2 \delta_{1}+2 \delta_{2}\right)+\delta_{1} \delta_{2}\right)\left(I_{1}^{*}+I_{2}^{*}\right)$

$$
\begin{gathered}
\frac{\mu \gamma_{2}\left(2 \mu^{2}+2 \mu \delta_{1}+2 \mu \delta_{2}+\delta_{1}{ }^{2}+\delta_{2}{ }^{2}\right)+\mu \gamma_{1}\left(\mu+\delta_{2}\right)^{2}\left(2 \mu+\delta_{1}\right)}{\left(\mu+\delta_{2}\right) \gamma_{1}+\gamma_{2}\left(\mu+\delta_{1}\right)} \\
\frac{\left(\mu+\delta_{2}\right)^{2} \gamma_{1}{ }^{2}+\mu\left(\mu+\delta_{1}\right)^{2} \gamma_{2}{ }^{2}+\mu \gamma_{2}\left(\mu+\delta_{1}\right)^{2}\left(2 \mu+\delta_{2}\right)}{\left(\mu+\delta_{2}\right) \gamma_{1}+\gamma_{2}\left(\mu+\delta_{1}\right)}
\end{gathered}
$$$$
b_{0}=\beta / N\left(\gamma_{1}+\gamma_{2}+\mu\right)\left(\mu+\delta_{2}\right)\left(\mu+\delta_{1}\right)\left(I_{1}^{*}+I_{2}^{*}\right)
$$

Thus, the values of $b_{2}, b_{1}$ and $b_{0}$ are positive. Furthermore

$$
\begin{gathered}
b_{3} b_{2} b_{1}-b_{1}^{2}-b_{3}^{2} b_{0}=b_{1}\left(b_{3} b_{2}-b_{1}\right)-b_{3}^{2} b_{0} \\
b_{1}\left(b_{3} b_{2}-b_{1}\right)= \\
b_{3} b_{2}-b_{1}=(\beta / N)^{2}\left(3 \mu+\delta_{1}+\delta_{2}+\gamma_{1}+\gamma_{2}\right)\left(I_{1}+I_{2}\right)^{2}+A A+B B \\
b_{3} b_{0}=\beta / N\left(\gamma_{1}+\gamma_{2}+\delta_{1}+\delta_{2}+4 \mu\right)\left(\gamma_{1}+\gamma_{2}+\mu\right)\left(\mu+\delta_{2}\right)\left(\mu+\delta_{1}\right)\left(I_{1}^{*}+I_{2}^{*}\right) \\
+(\beta / N)^{2}\left(\gamma_{1}+\gamma_{2}+\mu\right)\left(\mu+\delta_{2}\right)\left(\mu+\delta_{1}\right)\left(I_{1}^{*}+I_{2}^{*}\right)^{2}
\end{gathered}
$$

Since

$$
\begin{gathered}
\left(\gamma_{1}+\gamma_{2}+\mu\right)\left(\mu+\delta_{2}\right)\left(\mu+\delta_{1}\right)<\left(3 \mu+\delta_{1}+\delta_{2}+\gamma_{1}+\gamma_{2}\right) \\
\frac{\mu \gamma_{2}\left(2 \mu^{2}+2 \mu \delta_{1}+2 \mu \delta_{2}+\delta_{1}{ }^{2}+\delta_{2}{ }^{2}\right)+\mu \gamma_{1}\left(\mu+\delta_{2}\right)^{2}\left(2 \mu+\delta_{1}\right)}{\left(\mu+\delta_{2}\right) \gamma_{1}+\gamma_{2}\left(\mu+\delta_{1}\right)} \\
\frac{\left(\mu+\delta_{2}\right)^{2} \gamma_{1}{ }^{2}+\mu\left(\mu+\delta_{1}\right)^{2} \gamma_{2}{ }^{2}+\mu \gamma_{2}\left(\mu+\delta_{1}\right)^{2}\left(2 \mu+\delta_{2}\right)}{\left(\mu+\delta_{2}\right) \gamma_{1}+\gamma_{2}\left(\mu+\delta_{1}\right)}
\end{gathered}
$$

\section{Global Stability}

Theorem 6.1. If $R_{0}<1$, then $E_{0}$ is globally asymptotically stable.

\section{Proof:}

Define the Lyapunov function as follows:

$$
V_{1}=\left(\frac{\gamma_{1}\left(\mu+\delta_{2}\right)+\gamma_{2}\left(\mu+\delta_{1}\right)}{\mu+\gamma_{1}+\gamma_{2}}\right) E+\left(\mu+\delta_{2}\right) I_{1}+\left(\mu+\delta_{1}\right) I_{2}
$$

derivative $V_{1}$ with respect to time over the solution is:

$$
\begin{aligned}
\dot{V}_{1}= & \left(\frac{\gamma_{1}\left(\mu+\delta_{2}\right)+\gamma_{2}\left(\mu+\delta_{1}\right)}{\mu+\gamma_{1}+\gamma_{2}}\right)\left(\frac{\beta S}{N}\left(I_{1}+I_{2}\right)-\left(\mu+\gamma_{1}+\gamma_{2}\right) E\right) \\
& +\left(\mu+\delta_{2}\right)\left(\gamma_{1} E-\delta I_{1}-\mu I_{1}\right)+\left(\mu+\delta_{1}\right)\left(\gamma_{2} E-\delta I_{2}-\mu I_{2}\right)
\end{aligned}
$$

After some rearrangment, we have

$\dot{V}_{1}=\left(\frac{\beta S\left(\gamma_{1}\left(\mu+\delta_{2}\right)+\gamma_{2}\left(\mu+\delta_{1}\right)\right)}{N\left(\mu+\gamma_{1}+\gamma_{2}\right)}-1\right)\left(\mu+\delta_{1}\right)\left(\mu+\delta_{1}\right)\left(I_{1}+I_{2}\right)$

since $S \leq \Delta / \mu$, we have 


$$
\begin{gathered}
\dot{V} \leq\left(\frac{\Delta \beta\left(\gamma_{1}\left(\mu+\delta_{2}\right)+\gamma_{2}\left(\mu+\delta_{1}\right)\right)}{\mu N\left(\mu+\gamma_{1}+\gamma_{2}\right)}-1\right)\left(\mu+\delta_{1}\right)\left(\mu+\delta_{1}\right)\left(I_{1}+I_{2}\right) \\
\dot{V} \leq\left(R_{0}-1\right)\left(\mu+\delta_{1}\right)\left(\mu+\delta_{1}\right)\left(I_{1}+I_{2}\right)
\end{gathered}
$$

Clearly, if $R_{0}<1$, then $V^{\circ} \leq 0$. In case $V^{*}=0$, observe the largest invariant set contained in

$$
\begin{aligned}
\left\{X \in \Omega: V^{*}=\right. & 0\} \text { is } \Omega_{0}=\{X \in \Omega: I=0, I \\
& =(I 1, I 2)\}
\end{aligned}
$$

Since fact that $\Omega_{0}=D F E$ for $R_{0}<1$, the according to LaSalle-Lyapunov Theorem, as $\mathrm{t} \rightarrow \infty$, then all trajectories starting in $\Omega$ toward to DFE. This completes the proof.

Theorem 6.2. If $R_{0}>1$, then $E_{1}$ is globally asymptotically stable.

\section{Proof :}

Define Lyapunov function as follows :

$$
V_{2}=S-S^{*}-S^{*} \ln \frac{S}{S^{*}}+E-E^{*}-E^{*} \ln \frac{E}{E^{*}}+I_{1}-I_{1}^{*}-I_{1}^{*} \ln \frac{I_{1}}{I_{1}^{*}}+I_{2}-I_{2}^{*}-I_{2}^{*} \ln \frac{I_{2}}{I_{2}^{*}}
$$

Derivative $V_{2}$ with respect to time over the solution is :

$$
\begin{aligned}
\dot{V}_{2}=(\dot{S} & \left.-\frac{S^{*}}{S} \dot{S}\right)+\left(\dot{E}-\frac{E^{*}}{E} \dot{E}\right)+Q_{1}\left(\dot{I}_{1}-\frac{I_{1}^{*}}{I_{1}} \dot{I}_{1}\right)+Q_{2}\left(\dot{I}_{2}-\frac{I_{2}^{*}}{I_{2}} \dot{I}_{2}\right) \\
\dot{V}_{2}=( & \left.\Lambda-\frac{\beta S}{N}\left(I_{1}+I_{2}\right)-\mu S-\frac{S^{*}}{S} \Lambda+\frac{S^{*}}{S} \frac{\beta S}{N}\left(I_{1}+I_{2}\right)+\frac{S^{*}}{S} \mu S\right) \\
+ & \left(\frac{\beta S}{N}\left(I_{1}+I_{2}\right)-A E-\frac{E^{*}}{E} \frac{\beta S}{N}\left(I_{1}+I_{2}\right)+\frac{E^{*}}{E} A E\right) \\
+ & \left(Q_{1} \gamma_{1} E-Q_{1} B I_{1}-Q_{1} \frac{I_{1}^{*}}{I_{1}} \gamma_{1} E+Q_{1} \frac{I_{1}^{*}}{I_{1}} B I_{1}\right) \\
+ & \left(Q_{2} \gamma_{2} E-Q_{2} B I_{2}-Q_{2} \frac{I_{2}^{*}}{I_{2}} \gamma_{2} E+Q_{2} \frac{I_{2}^{*}}{I_{2}} B I_{2}\right)
\end{aligned}
$$

Next,

$$
\begin{gathered}
\dot{V}_{2}=\left(\Lambda-\mu S-\frac{S^{*}}{S} \Lambda+\frac{\beta S^{*}}{N}\left(I_{1}+I_{2}\right)+\mu S^{*}\right) \\
+\left(-A E-\frac{E^{*}}{E} \frac{\beta S}{N}\left(I_{1}+I_{2}\right)+A E^{*}\right) \\
+\left(Q_{1} \gamma_{1} E-Q_{1} B I_{1}-Q_{1} \frac{I_{1}^{*}}{I_{1}} \gamma_{1} E+Q_{1} B I_{1}^{*}\right) \\
+\left(Q_{2} \gamma_{2} E-Q_{2} C I_{2}-Q_{2} \frac{I_{2}^{*}}{I_{2}} \gamma_{2} E+Q_{2} C I_{2}^{*}\right)
\end{gathered}
$$

At equilibrium :

$$
\Lambda=\frac{\beta S^{*}}{N}\left(I_{1}^{*}+I_{2}^{*}\right)+\mu S^{*}
$$

Next,

$$
\begin{aligned}
\dot{V}_{2}=\left(\frac{\beta S^{*}}{N}\left(I_{1}^{*}+I_{2}^{*}\right)+\right. & \left.\mu S^{*}-\mu S-\frac{S^{*}}{S} \frac{\beta S^{*}}{N}\left(I_{1}^{*}+I_{2}^{*}\right)-\mu \frac{S^{* 2}}{S}+\frac{\beta S^{*}}{N}\left(I_{1}+I_{2}\right)+\mu S^{*}\right) \\
& +\left(-A E-\frac{E^{*}}{E} \frac{\beta S}{N}\left(I_{1}+I_{2}\right)+A E^{*}\right) \\
+ & \left(Q_{1} \gamma_{1} E-Q_{1} B I_{1}-Q_{1} \frac{I_{1}^{*}}{I_{1}} \gamma_{1} E+Q_{1} B I_{1}^{*}\right) \\
+ & \left(Q_{2} \gamma_{2} E-Q_{2} C I_{2}-Q_{2} \frac{I_{2}^{*}}{I_{2}} \gamma_{2} E+Q_{2} C I_{2}^{*}\right)
\end{aligned}
$$

$I_{1}$ And $I_{2}$ terms equating to zero, hence

$$
\begin{aligned}
& \left(\frac{\beta S^{*}}{N}-Q_{1} B\right) I_{1}=0 \\
& \left(\frac{\beta S^{*}}{N}-Q_{2} C\right) I_{2}=0
\end{aligned}
$$

then the solutions are

$$
Q_{1}=\frac{\beta S^{*}}{N B}, Q_{2}=\frac{\beta S^{*}}{N C}, A=Q_{1} \gamma_{1}+Q_{2} \gamma_{2}
$$

With

$$
\gamma_{1}=\frac{B I_{1}^{*}}{E^{*}}, \gamma_{2}=\frac{C I_{2}^{*}}{E^{*}}
$$

By substitute $Q_{1}$ and $Q_{2}$ and $A E^{*}=\beta S^{*}\left(I_{1}^{*}+I_{2}^{*}\right) / N$ , we get

$$
\left(Q_{1} \gamma_{1}+Q_{2} \gamma_{2}-A\right) E=0
$$

After some substitution and arrangements, we can rewrite the following derivative of Lyapunov function

$$
\begin{aligned}
\dot{V}=\frac{\beta S^{*}}{N}\left(I_{1}^{*}+I_{2}^{*}\right)+\mu S^{*}-\mu S-\frac{S^{*}}{S} & \frac{\beta S^{*}}{N}\left(I_{1}^{*}+I_{2}^{*}\right)-\mu \frac{S^{* 2}}{S}+\mu S^{*} \\
+-\frac{E^{*}}{E} \frac{\beta S}{N} I_{1}+ & -\frac{E^{*}}{E} \frac{\beta S}{N} I_{2}+\frac{\beta S^{*}}{B} I_{1}^{*}+\frac{\beta S^{*}}{N} I_{2}^{*} \\
& \quad-\frac{\beta S^{*}}{N} I_{1}^{*} I_{1}^{*} I_{1}^{*} E+\frac{\beta S^{*}}{N} I_{1}^{*}-\frac{\beta S^{*}}{N} \frac{I_{2}^{*}}{I_{2}} I_{2}^{*} E+\frac{\beta S^{*}}{N} I_{2}^{*}
\end{aligned}
$$

Or

$$
\begin{aligned}
\dot{V}=-\mu S^{*}\left(\frac{S}{S^{*}}+\frac{S^{*}}{S}-2\right)+\frac{\beta S^{*} I_{1}^{*}}{N}\left(3-\frac{S^{*} I_{1}}{S I_{1}^{*}}\right. & \left.-\frac{S E^{*} I_{1}}{S^{*} E I_{1}^{*}}-\frac{I_{1}^{*} E}{I_{1} E^{*}}\right) \\
& +\frac{\beta S^{*} I_{2}^{*}}{N}\left(3-\frac{S^{*} I_{2}}{S I_{2}^{*}}-\frac{S E^{*} I_{2}}{S^{*} E I_{2}^{*}}-\frac{I_{2}^{*} E}{I_{2} E^{*}}\right)
\end{aligned}
$$

When equilibirium $S=S^{*}, E=E^{*}, I_{1}=$ $I_{1}^{*}$, and $I_{2}=I_{2}^{*}$, we have $V^{*}=0$. By introducing function $g(x)=1-x-\ln (x)$ that monotone increasing for $x>0$, hence $g(x) \leq 0$. Then we can express inequalities as follows :

$$
\begin{gathered}
\mu S^{*}\left(2-\frac{S}{S^{*}}-\frac{S^{*}}{S}\right)=g\left(\frac{S}{S^{*}}\right)+g\left(\frac{S^{*}}{S}\right) \leq 0 \\
+\left(3-\frac{S^{*} I_{1}}{S I_{1}^{*}}-\frac{S E^{*} I_{1}}{S^{*} E I_{1}^{*}}-\frac{I_{1}^{*} E}{I_{1} E^{*}}\right)=g\left(\frac{S^{*} I_{1}}{S I_{1}^{*}}\right)+g\left(\frac{S E^{*} I_{1}}{S^{*} E I_{1}^{*}}\right)+g\left(\frac{E I_{1}^{*}}{E^{*} I_{1}^{*}}\right) \leq 0 \\
\left(3-\frac{S^{*} I_{2}}{S I_{2}^{*}}-\frac{S E^{*} I_{2}}{S^{*} E I_{2}^{*}}-\frac{I_{2}^{*} E}{I_{2} E^{*}}\right)=g\left(\frac{S^{*} I_{2}}{S I_{2}^{*}}\right)+g\left(\frac{S E^{*} I_{2}}{S^{*} E I_{2}^{*}}\right)+g\left(\frac{E I_{2}^{*}}{E^{*} I_{2}^{*}}\right) \leq 0
\end{gathered}
$$

so, for $R_{0}>1$, I follow that $V^{*} \leq 0$. Derivative Lyapunov function will $V^{\cdot}=0$ if only if

$S=S^{*}, E=E^{*}, I_{1}=I_{1}^{*}, I_{2}=I_{2}^{*}$. Therefore, we conclude that the largest compact invariance set $\left\{\left(S, E, I_{1}, I_{2}\right) \in \Omega \mid V^{*}=0\right\}$ id the singleton $E_{1}$. Hence LaSalle-Lyapunov Theorem implies that $E_{1}$ is globally asymptotically stable in $\Omega$.

\section{Conclusion}

In this paper, epidemic model of covid19 was rigorously analyzed. There are two equilibrium points, namely, disease free equilibrium and endemic equilibrium. The existance and the local stability of equilibria depends on $R_{0}$. Our analysis demonstrated that disease free equilibrium point is always locally asymptotically stable if $R_{0}<1$ and endemic equilibrium point always exists when $R_{0}>1$. Since the value of R0 depends on many factors such as infection rate $\beta$ and effectiveness of Quarantine $\delta-1$ Therefore, these intervention parameters need to be controlled in order to achieve a global stability of disease-free equilibrium.

\section{References}

1. https://www.worldometers.info/coronavirus/, Retrieved: 09-09-2021.

2. Corona Virus Disease (COVID-19)- Question and Answer. Available at https://www.who.int/emergencies/diseases/novel- 
coronavirus-2019/question-and-answers-hub/qadetail/q-a-coronaviruses [accessed Nopember, 2020]

3. Ceylan, Z. (2020). Estimation of COVID-19 prevalence in Italy, Spain, and France. Science of The Total Environment, 138817.

4. Chintalapudi, N., Battineni, G., and Amenta, F. (2020). COVID-19 disease outbreak forecasting of registered and recovered cases after sixty-day lockdown in Italy: A data driven model approach. Journal of Microbiology, Immunology and Infection.

5. Chatterjee, K., Chatterjee, K., Kumar, A., and Shankar, S. (2020). Healthcare impact of COVID19 epidemic in India: A stochastic mathematical model. Medical Journal Armed Forces India.

6. Jiao, J., Liu, Z., and Cai, S. (2020). Dynamics of an SEIR model with infectivity in incubation period and homestead-isolation on the susceptible. Applied Mathematics Letters, 106442.

7. Mandal, M., Jana, S., Nandi, S. K., Khatua, A., Adak, S., and Kar, T. K. (2020). A model-based study on the dynamics of COVID-19: Prediction and control. Chaos, Solitons \& Fractals, 109889.

8. He, S., Peng, and, and Sun, K. (2020). SEIR modeling of the COVID-19 and its dynamics. Nonlinear Dynamics, 101(3), 1667-1680.

9. Ivorra, B., Ferrez, M. R., Vela-Pz, M., and Ramos, A. M. (2020). Mathematical modeling of the spread of the coronavirus disease 2019 (COVID-19) taking into account the undetected infections. The case of China. Communications in nonlinear science and numerical simulation, 105303.

10. Aldila, D., Khoshnaw, S. H., Safitri, E., Anwar, Y. R., Bakry, A. R., Samiadji, B. M., ... and Salim, S.N. (2020). A mathematical study on the spread of COVID-19 considering social distancing and rapid assessment: The case of Jakarta, Indonesia. Chaos, Solitons \& Fractals, 110042. 